\title{
Human and Robotic Fish Interaction Controlled Using Hand Gesture Image Processing
}

\author{
Amarnathvarma Angani, Jin-Wook Lee, Teressa Talluri, \\ Jae-young Lee, and Kyoo Jae Shin ${ }^{*}$ \\ Department of ICT Creative Design, Busan University of Foreign Studies, \\ 65, Geumsaem-ro 485beon-gil, Geumjeong-gu, Busan 46234, Republic of Korea
}

(Received April 17, 2020; accepted August 11, 2020)

Keywords: aquarium robot, image sensors, biomimetic ornamental, hand gesture recognition, hand segmentation, human interactive control

This paper is about the control of robotic fish movement in an aquarium via human hand gestures detected by image sensors attached in the aquarium. In this study, sensors actively interact with humans and robotic fish. Image and radio frequency sensors are used to identify the position and color of robotic fish. Recently, we have studied human interactive control based on hand gesture recognition. Image sensors send the input signals of hand gestures obtained from real-time video images processed using tracking control algorithms, such as color mark, stop zone, and lead-lag tracking algorithms, to robotic fish. The movement of robotic fish is controlled via the movement of the two hands, where the left hand is for the fish to be controlled and the right hand is for controlling the movement of the robotic fish. Hand gesture recognition consists of hand feature segmentation and gesture recognition from the hand features. Our results show that interactive human control using hand gestures successfully controls the movement of robotic fish.

\section{Introduction}

In the fourth industrial revolution, computers have affected every aspect of life and various human activities. The development of human-computer interaction (HCI) to the next level will favorably impact human activities. Many researchers have been developing several robotic fishes. For example, Robot Tuna I was developed by the Massachusetts Institute of Technology (MIT) to improve autonomous underwater vehicles. ${ }^{(1)}$ Robot Pike of MIT attracts the interest of researchers because of its variable acceleration. (2) The manta ray robot of Evologics, Germany, can be applied to environmental monitoring, deep sea exploration, sensible ecological research, offshore industry, and marine security. ${ }^{(3)}$ Robotic koi, used to control the oxygen level in water, is advantageous in maintaining the health of fish. ${ }^{(4)}$ Researchers at the University of Essex developed robotic fish that can swim autonomously, similarly to real fish. ${ }^{(5)}$ PPF-04 was developed by Koichi Hirata of National Maritime Research Institute (NMRI) to improve

*Corresponding author: e-mail: kyoojae@bufs.ac.kr https://doi.org/10.18494/SAM.2020.2925 
the movements and performance of underwater robots such as boats, ships, submarines, and underwater craft. ${ }^{(6)}$ This robotic fish can swim either by moving its body or fin or by median and paired fin (MPF) propulsion. ${ }^{(7)}$

Nowadays, robotic fishes are becoming increasingly popular as a tool for realizing automatic operation. $^{(8)}$ Further development of Human Computer Interaction (HCI) will increasingly support human activities. The control of robotic fish with hand gestures can be realized by utilizing image sensors to analyze video frames. The background in the video frames is removed. Contours, convex points, defect points, and a convex hull are extracted from the video frames. The convex hull is used to identify hand gestures. Gestures are an easy way to interact with computers. The human hand is a highly deformable articulated object with many degrees of freedom, and its different postures and movements can be used for expressing information for various purposes.

In our research, we also focused on the design of the robotic fish for a subaqueous system based on the structure of the fish domi (Pagrus major), which lives in the ocean off South Korea. Robotic domi fishes were exhibited at the 2012 International YEOSU Expo, and their performance was excellent. These robotic fishes were made by Science Robotic Company (SRC) and Artificial Intelligence Robot (AIRO) in South Korea.

Chen et al. ${ }^{(9)}$ proposed a recognition system with a hidden Markov model (HMM) training mechanism for recognizing hand gestures in a stationary background. The research of many researchers has been limited to merely a hand gesture recognition system without implementing it in a control system. Our method of controlling a robotic fish with hand gestures is the first of its kind in the world.

In this work, we developed a hand gesture recognition system for controlling the movement of robotic fish via hand gestures. Human-robot interaction (HRI) is an emerging field of study dedicated to understanding, designing, and evaluating robotic systems for use by humans. HRI is defined as requiring communication between robots and humans. We adopted two image processing technologies for identifying the color of robotic fish and human gestures using the OpenCV (Computer Vision) algorithm. A user will be able to control any robotic fish in an aquarium using hand gestures. The right hand is for controlling the movement of the fish and the left hand is for controlling a specific fish. Two image sensors are used, one to collect human hand gestures and the other to collect the robotic fish position in the aquarium. The user can select the tracking control algorithm, such as the color mark, stop zone, or lead-lag tracking control algorithm.

In this study, sensors are the major tools of active communication between a human and a robot, for example, image sensors to detect the movements of the robotic fish and the human hand gestures, and the radio-frequency (RF) module for receiving signals from the human and transmitting data to the robot. Users can control the movement of robotic fish by using their two hands where the left hand controls a specific robotic fish and the right hand controls the native movement of the robotic fish. Real-time video images of hand gestures are processed to convert them to data that is transmitted to the robotic fish. Our results show that our interactive human control system with hand gesture processing using tracking control algorithms and image sensors successfully controls the movement of robotic fish. 


\section{Design of Human-Robotic Fish Interactive Control System}

Human gestures include the movements of the body, face, and hands. With hand and arm gesticulation, $90 \%$ of gestured communication is possible. ${ }^{(10)}$ Communication and interaction are broadly divided into two general categories: remote and proximate. Our system first recognizes and tracks the human hand and fingertips in a video sequence in advance. Then, the posture of the hand and the positions of the fingers are computed using Euclidean distances of the fingertips to determine the movement between consecutive frames of the video sequence using hand segmentation, contour extraction, a convex hull, and convexity defects.

The proposed human-robotic fish interactive control system shown in Fig. 1 consists of the robotic fish, image sensors (cameras), aquarium, RF transmitter (RF-TX), RF receiver (RF-RX), and human hand gestures. In this work, sensors play the important role of receiving signals of human hand movement and transmitting those signals to robotic fish such that they will move in accordance with the human hand gesture.

Figure 1(a) shows the human-robotic fish interaction, which was explained in the previous paper entitled "Human Interaction Control of the Robotic Fish Using Detecting Object Algorithms". (11) How human hand gestures are used to control the robotic fish in the aquarium were explained in that paper. The main concept of those algorithms was that all the robotic fishes approach a human being standing in front of the aquarium. Figure 1(b) illustrates the concept of the proposed human-robotic fish interactive control via human hand gestures. The following are the two main tasks: the control of fish movement and the selection of different algorithms, investigated in the current work.

Case (1): Users can control the movement of the robotic fish by gesturing with the left and right hands, where the left hand controls a specific fish and the right hand controls the native movement of the fish, that is, left, right, up, down, or forward movement, in the aquarium.

Case (2): Through specific human hand-finger gestures, users can select different tracking control algorithms, such as color mark, stop zone, and lead-lag tracking control algorithms. For example, to control the color mark tracking control algorithm, the user raises one finger of either the left or right hand. Here, the right hand is used

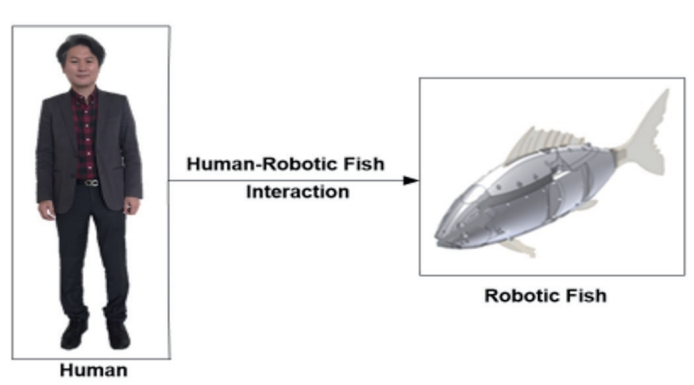

(a)

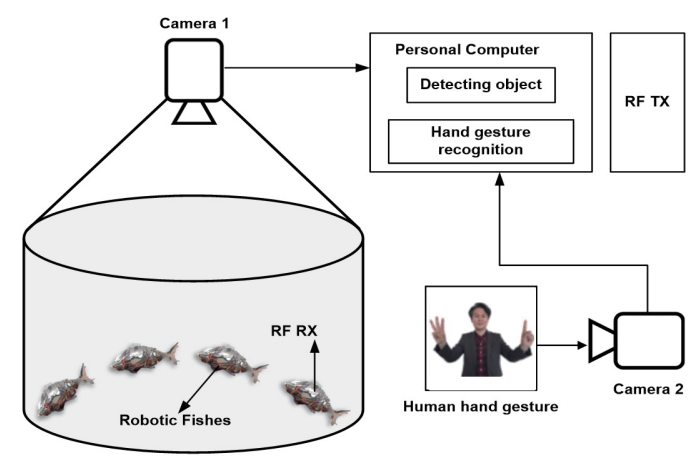

(b)

Fig. 1. (Color online) (a) Human-robotic fish interaction. (b) Proposed aquarium in which robotic fish can be controlled by human hand gestures. 
to control the robotic fish in the aquarium. If the user wants to use the stop zone tracking control algorithm, two fingers are raised, and to use the lead-lag tracking control algorithm, three fingers are raised.

Each robotic fish body is designed with an optimized inner area to mimic the structure of a real fish. The dynamic forces of the robot are determined from its instantaneous swimming movement in the aquarium. The forces acting on the robotic fish are the thrust force in the forward direction along the $x$-axis, water resistance in the reverse direction along the $y$-axis, and gravity in the vertical direction along the $z$-axis, as shown in Fig. 2(a).

The mathematical modeling of the buoyancy force acting on the fish in the aquatic environment is expressed as the fixed coordinate system $\left[\hat{x}_{i}, \hat{y}_{i}, \hat{z}_{i}\right]$ and the swimming Eq. (1) of robotic fish is a continuous function of a discrete function of the kinematic streamer model taken from the analysis proposed by Lighthill.

$$
\theta_{i}=a_{i} \sin \left(2 \pi f t+p_{i}\right)
$$

Here, $\theta_{i}$ is the joint angle, $a_{i}$ is the amplitude, $f$ is the frequency, $t$ is time, and $p_{i}$ is the phase difference between heaving and pitching movements. Then, the sliding frequency of the robotic fish swimming in the water can be determined using the Lighthill analysis. To alter the movement of the robotic fish, for example, for up and down swimming, the robot is designed to move back and forth relative to its center of gravity similar to the gravity principle of biomimetic fish. ${ }^{(12)}$ The proposed system will be controlled by the human hand-finger gestures.

\section{Modeling of Human Interactive Control Using Hand Gesture Recognition}

\subsection{Hand gesture recognition}

\subsubsection{Hand segmentation}

The detection of the hand gesture in this project is based on the segmentation process. Segmentation is the process of grouping points that belong to the same object into segments. The main idea is to extract a set of points from an image. ${ }^{(12)}$ The steps of the segmentation process are explained below.

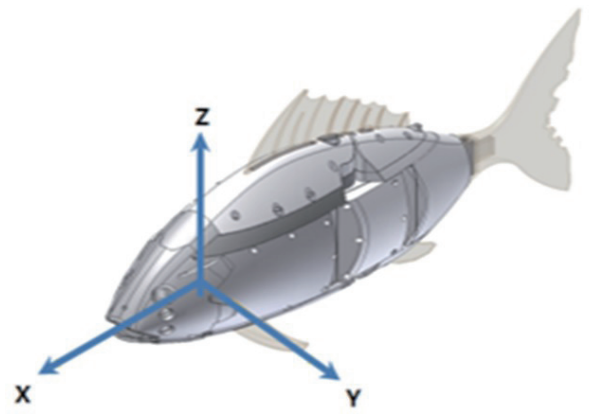

(a)

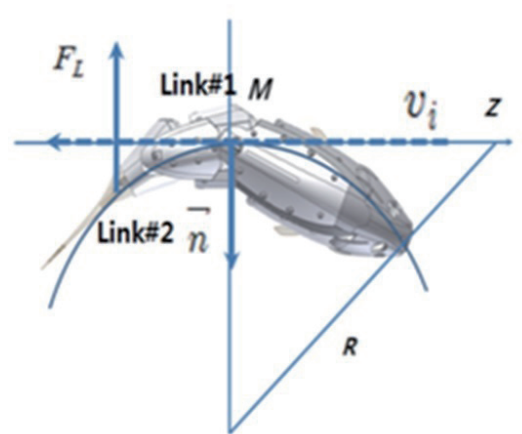

(b)

Fig. 2. (Color online) (a) Axial direction of robotic fish. (b) Lift and swimming rotation in the aquarium. 
(a) Capture of target object and recording of frames: The initial step is the capture of the target object and recording of frames to detect the background of the target object. To accomplish this, the background should be white to avoid noise and achieve the optimal extraction of the target object. ${ }^{(13)}$

(b) Extraction of the region of interest (ROI): The following classical method is used to extract the target object or frame from video after capturing. The relevant frames are converted to grayscale and the Gaussian blur process is applied to delete noise. The initial background subtraction entails a reference frame from which each new frame is subtracted. The result, which is a binary (black and white) image, is thresholded to highlight the region of nonstatic objects. ${ }^{(14)}$

(c) Contour extraction: A contour map comprises contour lines that show the steepness of slopes and the valleys and hills. The slope or gradient function is always perpendicular to the contour lines. When the lines are close together, the magnitude of the gradient is large. Contours are straight lines or curves representing the intersection of one or more horizontal planes with a real or hypothetical surface..$^{(15)}$

(d) Drawing of contours: A contour line is drawn around the white blob of the hand obtained by thresholding the input image. There may be the possibility that more than one blob will be formed in the image owing to noise in the background. Therefore, the contour lines are also drawn on the smaller white blobs. All blobs due to noise are considered to be small; thus, the large contour is deemed to be that of the hand and is considered for further processing. The vector contains a set of contour points in the coordinate form as

$$
E_{\text {shank }}=E_{\text {stretch }}+E_{\text {bend }}+E_{\text {line }}+E_{\text {edge }}
$$

where $E_{\text {stretch }}=\int k(s)\left\|X_{s}(s)\right\|^{2} d s, E_{\text {bend }}=\int \lambda(s)\left\|X_{s s}(s)\right\|^{2} d s, E_{\text {edge }}=-\int \mu(s)\|\operatorname{grad} I\|^{2} d s$, and $E_{\text {line }}=\int(\lambda(s)+\kappa(s))\left(\left|X_{s}(s)\right|^{2}+\left|X_{s s}(s)\right|^{2}\right) d s$.

\subsubsection{Hand recognition features}

(a) Convex points: Convex points are the points in the convex sets in the Euclidean space. For example, when a set of points is a bounded subset of the plane, the convex hull can be visualized as the shape formed by a rubber band stretched around this set of points. These points are the convex points.

(b) Defect points: Defect points are the points used to distinguish the fingers from the palm of the hand. This detection process depends on four points: the start point, end point, farthest point, and approximate distance to the farthest point. The line joining the start and end points traces the hand.

(c) Convex hull: The convex hull of a set of points in the Euclidean space is the smallest convex set that contains the entire set of given points. The convex hull is drawn around the contour of the hand such that all contour points are within the convex hull. This generates an envelope around the hand contour. The convex hull of a set of points $S$ in $n$ dimensions is the intersection of all convex sets containing $S$. For $N$ points $p_{1}, \ldots, p_{N}$, the convex hull $C$ is then given by 


$$
C(s)=\left\{\sum_{j=1}^{N} \lambda_{j} p_{j}: \lambda_{j} \geq 0 \text { for all } j \text { and } \sum_{j=1}^{N} \lambda_{j}=1\right\},
$$

where $C(s)$ is the set of all convex combinations of its points, $p_{j}$ indicates each point, and $\lambda_{j}$ is the coefficient of points (obtained from their position coordinates)

(d) Convexity defects: Both convexity and defect points form the convex hull that joins the points used to draw the green outline of the hand. The set of contour points of the hand fits within the hull when the convex hull is drawn around the contour of the hand. The minimum number of points is used to form the hull and the property of convexity is maintained. From the hull formation and convexity properties, the finger can be distinguished and are used to track the hand. When the hand moves, the robotic fish follows the hand and fingers.

\subsection{Human interactive control using hand gesture recognition}

In general, the color of an object depends on the characteristics of the perceiving eye and brain. Physically, the color of objects is perceived from the light that is reflected from their surfaces. In the aquarium, differently colored robotic fish mimic actual fish. The setup for human interactive control with hand gesture consists of a camera, an OpenCV library, and color detection and position data. Here, we used the OpenCV library along with the python programming language. When the camera is interfaced with raspberry Pi through the OpenCV library, it can identify the color of the robotic fish on the basis of the hue, saturation, and value (HSV) color space and obtain position data of the robotic fish in the aquarium.

The properties of object detection algorithm ${ }^{(16-21)}$ are shown in Table 1. The color segment algorithm is suited for noise reduction and object detection by the current system. With our proposed system, we are approaching a new application area of human interactive control. Figure 3 shows the hand gesture recognition system that we followed based on the number of fingers opened or closed. The flowchart of human interactive control is given in Fig. 4. The detailed description about the working of this hand gesture and execution of the algorithm is explained in the subsequent sections.

All robotic fishes are swimming in the aquarium. At the position of the color mark, the robotic fish that is in this color mark zone will stop. When the user deactivates the color mark, the robotic fish swims together with other robotic fishes as before. The robotic fishes are controlled via signals from the RF modem. ${ }^{(22-27)}$

Table 1

Properties of object detection algorithms.

\begin{tabular}{lc}
\hline Parameter & Color segment algorithm \\
\hline Object detection & Color threshold value depends on color \\
Threshold & Color \\
Variability & Detects each object \\
Position data & Good \\
Identification of object & Simple \\
Noise & None \\
Accuracy & Stable \\
Tracking & Possible \\
\hline
\end{tabular}




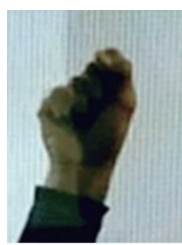

(a)

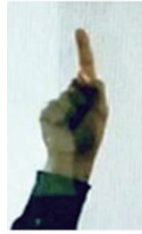

(d)

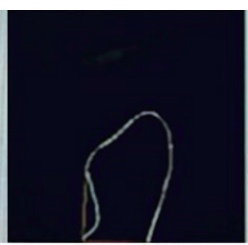

(b)

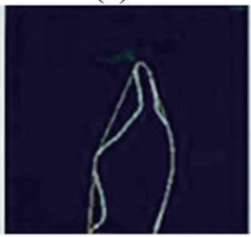

(e)

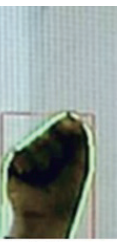

(c)

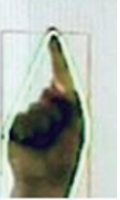

(f)

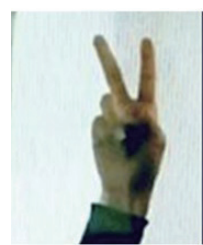

(g)

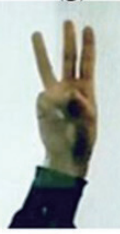

(j)

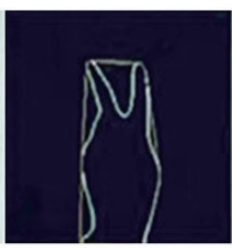

(h)

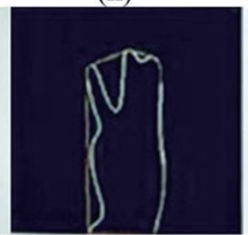

(k)

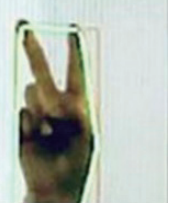

(i)

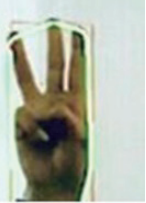

(l)

Fig. 3. (Color online) Human hand gesture recognition: (a) original null hand gesture, (b) threshold image, (c) no algorithm is selected, (d) original one-finger hand gesture, (e) threshold image of one finger, (f) color mark tracking control algorithm is selected, (g) two-finger hand gesture, (h) threshold image of two fingers, (i) stop zone tracking control algorithm is selected, (j) three-finger hand gesture, (k) threshold image, and (l) lead-lag tracking control algorithm is selected.

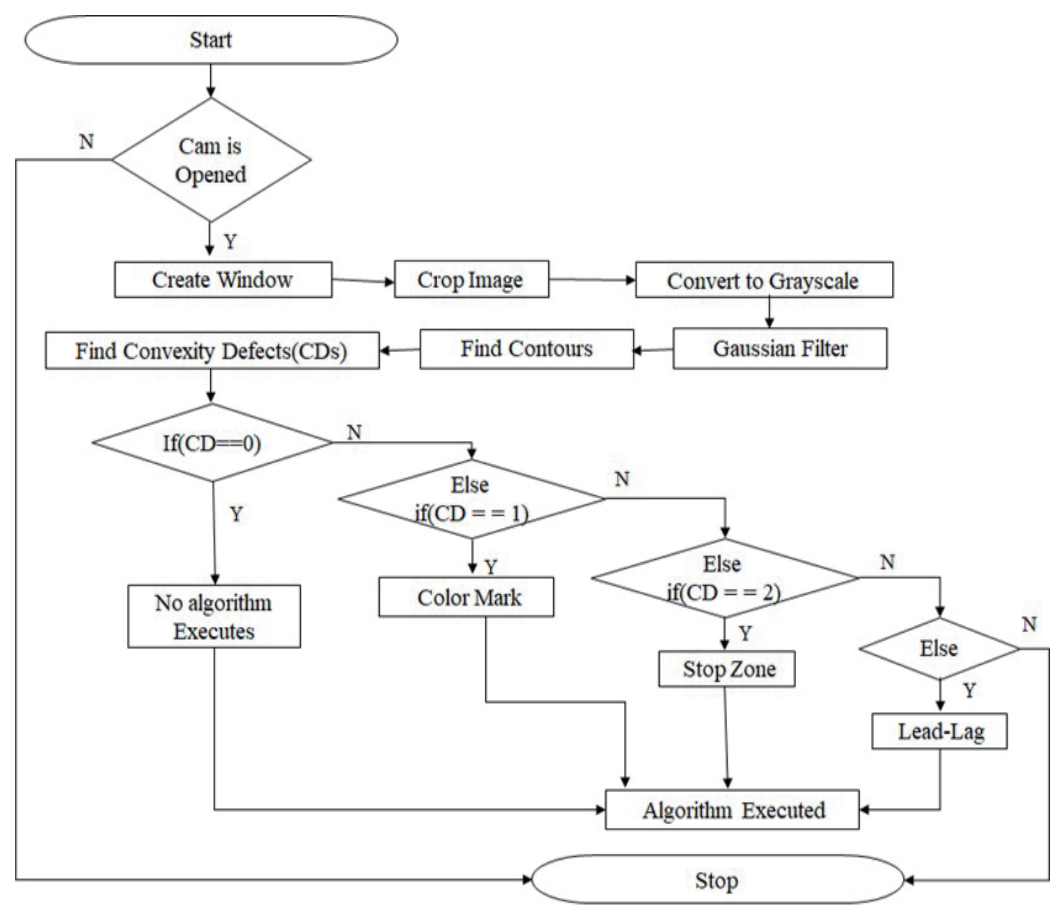

Fig. 4. Flowchart of human interactive control.

\section{Experiment Results}

\subsection{Proposed experimental system of HRI}

The proposed experimental system consists of an aquarium, robotic fishes, raspberry Pi, cameras, RF TX, object-detection algorithm (color segment algorithm), and human interactive control algorithm for hand-finger gestures, as shown in Fig. 5. 


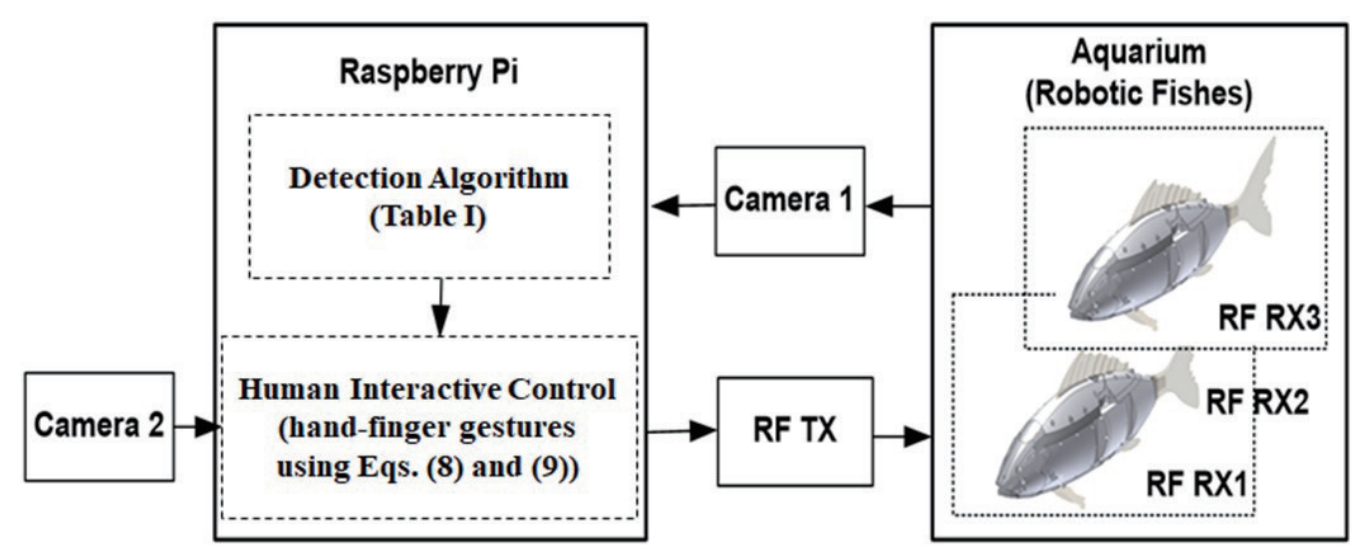

Fig. 5. Experimental configuration of proposed human interactive control.

\subsection{Control of robotic fish using hand-gesture recognition with RF modem}

The results of robotic fish control in the aquarium are shown in Figs. 6-9. When the user raises one finger, the algorithm is switched automatically to the color mark tracking control. The results show that all the robotic fishes are stopped when the color mark is green, blue, and yellow then the position coordinate value is $(361,401)$.

\subsection{Results of human interactive hand control with hand gesture recognition}

The results shown in Fig. 9 indicate that the robotic fishes stop at the color mark and resume autonomous swimming when the mark is removed. The results of color mark tracking analysis are shown in Fig. 7. Color marks were placed at two positions in the aquarium. When the user removed the color marks, the robotic fishes resumed autonomous swimming.

When the user raised two fingers, the algorithm was switched automatically to the stop zone tracking control algorithm. All the robotic fishes stopped at the position $(501,242)$ in the aquarium. This position command was given through the programming in the algorithm. The blue, red, and yellow robotic fishes have position coordinate values of $(561,294),(545,317)$, and $(526,373)$ respectively. When the robotic fishes leave this zone, they resume autonomous swimming.

The stop zone tracking analysis is shown in Fig. 9. One stop zone is designated in the aquarium. When robotic fishes reach this stop zone, they stop and swim at this position automatically until they cross the stop zone boundary. Then, these robotic fishes resume swimming autonomously. When the user raised three fingers, the algorithm was switched automatically to the lead-lag tracking control algorithm (Fig. 8). Two robotic fishes in the aquarium stopped swimming. This algorithm is based on the distance between two robotic fishes, for instance, the lead red robotic fish is at position $(518,378)$ and the lagging yellow robotic fish is at position $(549,251)$. If the distance between the two robotic fishes is greater 


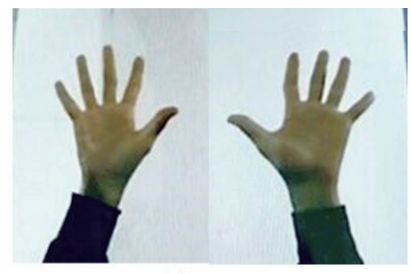

(a)

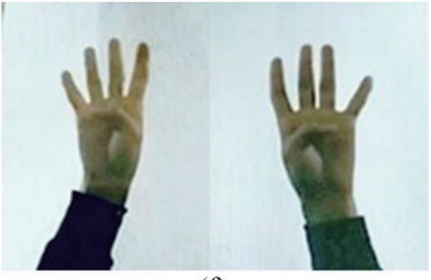

(f)

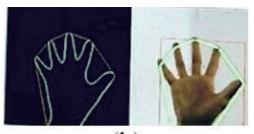

(b)

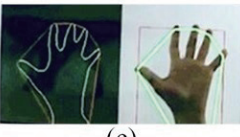

(c)

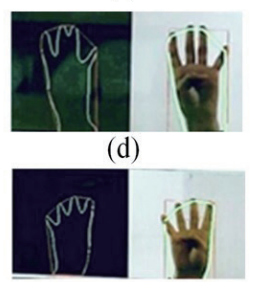

(e)

Fig. 6. (Color online) Hand-finger gestures. (a) Five-finger hand gesture. (b) Right hand: robotic fish ID is five. (c) Left hand: robotic fish movement is five (forward). (d) Left hand: robotic fish ID is four. (e) Right hand: robotic fish movement is four (to the right). (f) Four-finger hand gesture.

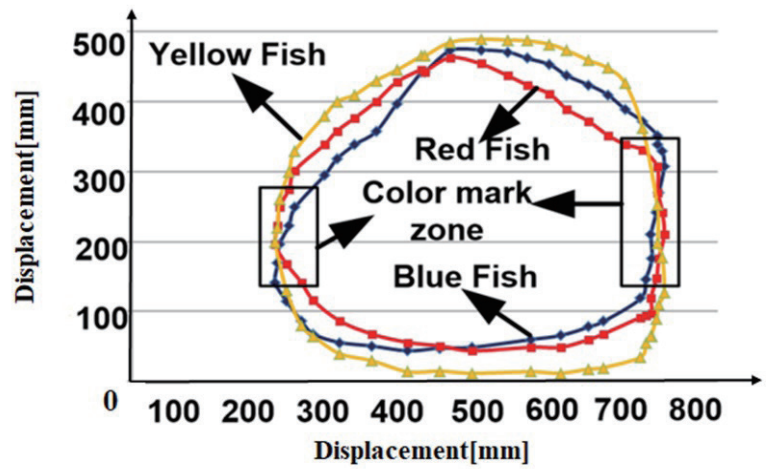

Fig. 7. (Color online) Results of analysis of color mark tracking control.

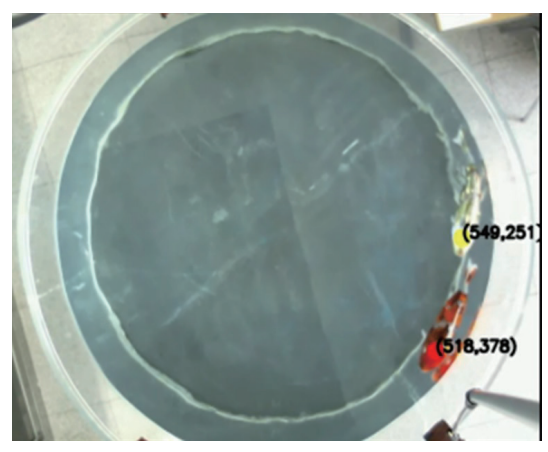

(a)

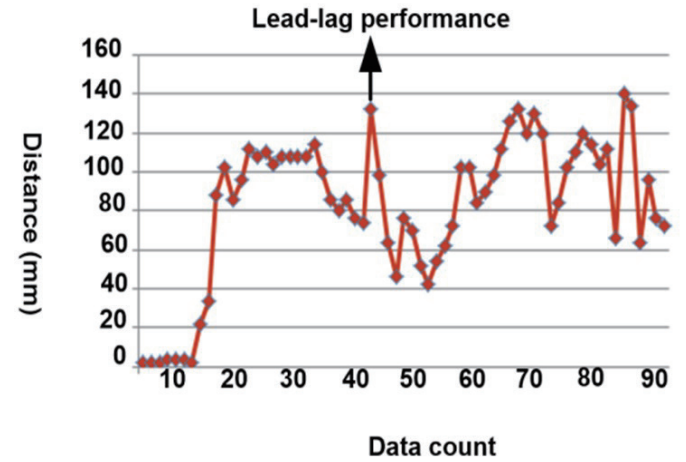

(b)

Fig. 8. (Color online) (a) Results of lead-lag tracking control by three-finger hand gesture and (b) analysis results of lead-lag tracking control.

than $150 \mathrm{~mm}$, the lead robotic fish will wait for the lagging robotic fish to reach it, after which they swim together. This process continues until this algorithm is stopped.

The results of lead-lag tracking control analysis of two robotic fishes are shown in Fig. 9. Robotic fish 1 is the red robotic fish and robotic fish 2 is the yellow robotic fish. The position of robotic fish $1\left(x_{1}, y_{1}\right)$ is $(518,378)$ and that of robotic fish $2\left(x_{2}, y_{2}\right)$ is $(549,251)$, as shown in Fig. 9(a). Then, using these position coordinates, we determined the distance between the two robotic fishes and analyzed the performance of lead-lag tracking distance control of the robotic fishes. The performance test of this algorithm yielded satisfactory results. 


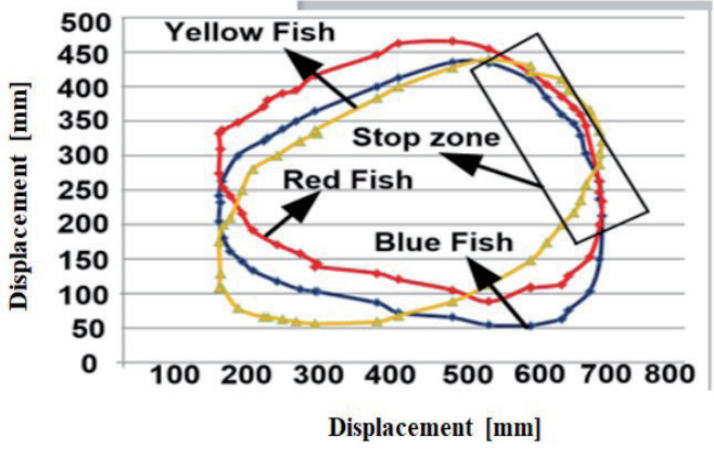

(a)

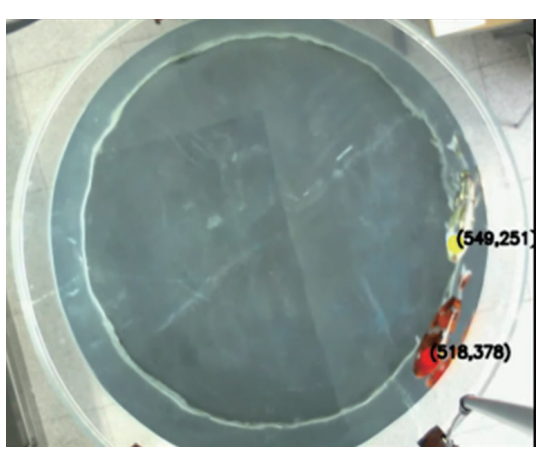

(b)

Fig. 9. (Color online) (a) Analysis results of stop zone tracking control and (b) results of stop zone tracking control using two-finger hand gesture.

\section{Conclusions}

We presented a new method of controlling robotic fish using hand gestures. This method is based on data obtained by sensors; hence, sensors are an important component in this system. For example, image sensors and RF module sensors are used in the control of the movement of robotic fish using two hands and for wireless communication, respectively. In the experiment, the user utilized hand gestures to select the tracking control algorithm from among the color mark, stop zone, and lead-lag tracking control algorithms. Hand segmentation and gesture recognition from the hand features were performed to generate control signals. In this work, raspberry Pi was used instead of a personal computer. The performance test of these algorithms yielded satisfactory results. In the future, research work to realize human interactive control of 3D hologram fish will be carried out.

\section{Acknowledgments}

This work was supported by the Korea Ministry of Trade, Industry and Energy under a grant of "Design Expert Training for Factory Automatic of the Based ICT Energy".

\section{References}

1 M. Anderson and K. N. Chhabra: Integr. Comp. Biol. 42 (2002) 118. https://doi.org/10.1093/icb /42.1.118

2 J. M. Kumph: Maneuvering of a Robotic Pike (Massachusetts Institute of Technology, USA, 2000). http://hdl. handle.net/1721.1/8968

3 Z. Chen, T. I. Um, and H. B. Smith: Int. J. Smart Nano Mater. 3 (2012) 296. https://doi.org/10.1080/19475411.20 12.686458

4 A. A. Sequeira, A. Usman, O. P. Tharakan, and M. Z. Ali: Int. J. Autom. Mechatronics Robot. 3 (2016) 106.

5 H. Hu: Proc. 2006 IEEE SMC UK-RI Chapter Conf. (IEEE, 2006).

6 K. Hirata, T. Takimoto, and K. Tamura: 1st Int. Symp. Aqua Bio-mechanisms. https://pdfs.semanticscholar. org/221a/c2b05ad2fb8acadf66b9868f18832e0dff98.pdf (accessed 2000).

7 M. Sfakiotakis, D. M. Lane, and J. B. C. Davies: IEEE J. Oceanic Eng. 24 (1999) 237. https://doi. org $/ 10.1109 / 48.757275$

8 K. J. Shin: KIPS Trans. Software Data Eng. 4 (2015) 219. https://doi.org/10.3745/KTSDE.2015.4.5.219 
9 F. S. Chen, C. M. Fu, and C. L. Huang: Image Vision Comput. 21 (2003) 745. https://doi.org/10.1016/S02628856(03)00070-2.

10 S. Mitra and T. Acharya: IEEE Trans. Syst. Man Cybern. Part C Appl. Rev. 37 (2007) 311. https://doi. org/10.1109/TSMCC.2007.893280

11 B. J. Kim and K. J. Shin: IEIE Trans. Smart Process. Comput. 7 (2018) 279. https://doi.org/ 10.5573/ IEIESPC.2018.7.4.279

12 M.Y. Rao, G. L. Chowdary, and K. J. Shin: Proc. 2016 KIIT Conf. http://www.dbpia.co.kr/pdf/pdfView. do?nodeId=NODE06690912

13 K. J. Shin and M. Y. Rao: Int. Conf. Electronics, Information, and Communications (ICEIC, IEEE, 2016). https://doi.org/10.1109/ELINFOCOM.2016.7562987.

14 W. K. Pratt: Introduction to Digital Image Processing (CRC Press, California, 2013).

15 T. Bose and M. Francois: Digital Signal and Image Processing (Wiley, US, 2003).

16 K. J Shin and M. Y. Rao: Int. J. Trend Res. Develop. 3 (2016) 312. http://www.ijtrd.com/papers/IJTRD4260. pdf

17 K. J. Shin and Y. M. Rao: Proc. 2016 Future Technologies Conf. (IEEE, 2016). https://doi.org/10.1109/ FTC.2016.7821712

18 K. J. Shin: J. Current Res. 8 (2016) 41012. https://pdfs.semanticscholar.org/f6f8/ d6384212479eb8d97dccdc4cf9627100a875.pdf

19 K. J. Shin: Future Technologies Conf. (IEEE, 2016). https://doi.org/10.1109/FTC.2016.7821709

20 M. Akbar and K. J. Shin: Proc. 2016 Korea Information Processing Society Conf. (2016). https://doi. org/10.3745/PKIPS.y2016m10a.510

21 G. G. Bueno: Learning Image Processing with OpenCV (Packt Publishing Ltd., United Kingdom, 2015).

22 K. J. Shin: IEIE Trans. Smart Process. Comput. 5 (2016) 375. https://doi.org/10.5573/IEIESPC.2016.5.6.375

23 S. H. Kang, T. U. Kang, A. V. Angani, and J. B. Lee: Int. Conf. Architecture, Construction, Environment and Hydraulics. (IEEE, 2019) 21. 10.1109/ICACEH48424.2019.9041845

24 E. S. Kim, C. B. Kim, T. Teressa, A. Angani, and K. J. Shin: Proc. The Institute of Electronics and Information Engineers of Korea 2019 Summer Conf. (2019) 817.

25 A. V. Angani, J. H. Lee, and K. J. Shin: Proc. 2015 Korea Information Processing Society Conf. (2015) 13541357.

26 A. V. Angani, S. H. Kang, T. Teressa, T. U. Kang, M. J. Seo, and K. J. Shin: Sens. Mater. 32 (2020) 3491. https://doi.org/10.18494/SAM.2020.2926

27 A. V. Angani, T. Teressa, J. W. Lee, E. S. Kim, and J. B. Lee: Sens. Mater. 32 (2020) 3507. https://doi. org/10.18494/SAM.2020.2927

\section{About the Authors}

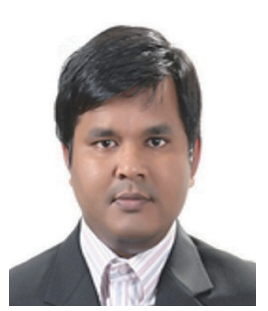

Amarnathvarma Angani is a Ph.D. student under Professor Kyoo Jae Shin of ICT Creative Design at the Busan University of Foreign Studies (BUFS), Busan, South Korea. He received his M.S. degree also from BUFS in 2018, and his B.Tech. degree in mechanical engineering (Mech) in 2014 from Jawaharlal Nehru Technological University, Kakinada (JNTUK), India. He received his diploma in automobile engineering in 2007 from Andhra Polytechnic, Kakinada, India. He has four years of experience as a design engineer at Infotech Enterprises (2007 to 2011). He is conducting research on ICT vertical aquarium fish farms, fish robots, and 3D holograms. He is interested in intelligent robots, image processing, and smart farms and aquariums fabricated using new energy and IoT technology 


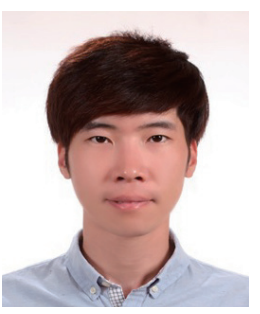

Jin-Wook Lee is pursuing his M.S. degree in the Department of ICT Creative Design at Busan University of Foreign Studies (BUFS) Busan, South Korea. He graduated from the Department of Computer Engineering at Pusan National University. He is studying marine IOT and developing unmanned aerial vehicle technology.

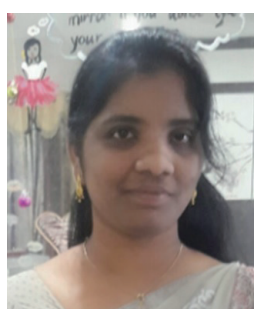

Teressa Talluri is pursuing her Ph.D. degree in the Department of ICT Creative Design at Busan University of Foreign Studies (BUFS) Busan, South Korea. She received her M.S. degree in thermal engineering from Jawaharlal Nehru Technological University (JNTUK) Kakinada, India, in 2014.

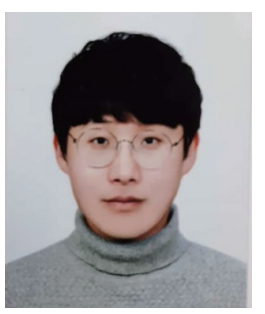

Jae-young Lee is pursuing his Ph.D. degree in the Department of ICT Creative Design at Busan University of Foreign Studies (BUFS) Busan, South Korea. He received his M.S. degree in robotics engineering at Busan University of Foreign Studies (BUFS) Busan, South Korea, in 2019. He received his diploma in elementary school education and computer education in 2011 from Busan National University of Education (BNUE). He is conducting research on smart fish farm controlling systems with artificial intelligence and IoT technology. He is also interested in image processing and intelligent robots.

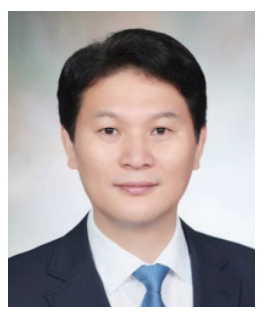

Kyoo Jae Shin is a professor in the Department of Intelligence Robot Science at the Busan University of Foreign Studies (BUFS), Busan, South Korea. $\mathrm{He}$ is the director of Future Creative Science Research Institute at BUFS. He received his B.S. degree in electronics engineering in 1985 and M.S. degree in electrical engineering in 1988 from Cheonbuk National University (CNU) and his Ph.D. degree in electrical science from the Pusan National University in 2009. Dr. Shin has served as a professor at the Navy Technical Education School and as the main director of research associates of the dynamic stabilization system in the Busan Defense Weapon Research Institute. He has also researched and developed a fish robot, submarine robot, automatic milking robot with manipulators, personal electrical vehicle, smart accumulated aquarium using heat pump, solar tracking system, 3D hologram system, and gun/turret stabilization system. He is interested in intelligent robots, image signal processing application systems, and smart farms and aquariums fabricated using new energy and IoT technology. 